\title{
NUCLEAR CHARGE RADII FROM X-RAY TRANSITIONS IN MUONIC ATOMS OF CARBON, NITROGEN AND OXYGEN
}

\author{
T. DUBLER, L. SCHELLENBERG and H. SCHNEUWLY \\ Institut de Physique, Université de Fribourg, 1700 Fribourg, Switzerland
}

R. ENGFER, J. L. VUILLEUMIER, H. K. WALTER and A. ZEHNDER

Laboratorium für Hochenergiephysik der ETH Zürich, 5234 Villigen, Switzerland

and

B. FRICKE

Gesellschaft für Schwerionenforschung mbH, Darmstadt, Germany

Received 1 November 1973

Abstract: Energies of muonic X-rays of the $K$-series of carbon, nitrogen and oxygen have been measured with an accuracy of about $15 \mathrm{eV}$. Root mean square radii of the nuclear charge distributions were deduced. The results $2.49 \pm 0.05 \mathrm{fm}$ for carbon, $2.55 \pm 0.03 \mathrm{fm}$ for nitrogen and $2.71 \pm 0.02 \mathrm{fm}$ for oxygen are in good agreement at comparable accuracy with recent electron scattering data.

NUCLEAR REACTIONS muonic atoms ${ }^{12} \mathrm{C},{ }^{14} \mathrm{~N},{ }^{16} \mathrm{O},{ }^{103} \mathrm{Rh}(\mu, 2 \mathrm{n} v)^{101} \mathrm{Ru}$; $E$ approx 0 ; measured muonic X-rays, $E_{\gamma},{ }^{12} \mathrm{C},{ }^{14} \mathrm{~N},{ }^{16} \mathrm{O}$ deduced nuclear charge radius, ${ }^{101} \mathrm{Ru}$ déduced transitions.

\section{Introduction}

Energies of muonic X-rays permit an almost model-independent determination of nuclear root mean square ( $\mathrm{rms}$ ) radii for light nuclei. These radii can be compared to those obtained from elastic electron scattering experiments, provided the scattering data have been measured at an equivalent momentum transfer $q_{\text {eq }}$. Nuclear charge radii, determined from scattering cross sections at this momentum transfer show nearly the same model dependence compared to those obtained from $\mu \mathrm{X}$-ray energies ${ }^{1}$ ). A representative value for $Z=20$ is $q_{\mathrm{eq}} \approx 0.3 \mathrm{fm}^{-1}$ corresponding to an energy of $50 \mathrm{MeV}$ and a scattering angle of $72^{\circ} ; q_{\mathrm{eq}}$ is a slowly varying function of $Z$.

Any difference in radii obtained by the two methods could be attributed to a difference in the size of the muon and electron or to a difference between the muonic and electronic effective interaction ${ }^{2}$ ). For the difference between the muon and electron radii a limit of $\left\langle r^{2}\right\rangle_{\mu}-\left\langle r^{2}\right\rangle_{\mathrm{e}}<0.014 \mathrm{fm}$ has been derived ${ }^{3}$ ) from comparison between high-energy $\mathrm{e}-\mathrm{p}$ and $\mu-\mathrm{p}$ scattering experiments; the results of the $g-2$ experiment places a limit on the muon mean square radius of 
$\left\langle r^{2}\right\rangle_{\mu}<0.004 \mathrm{fm}$ [refs. $\left.\left.{ }^{4,5}\right)\right]$. These limits are too small to be seen in a comparison of $\mu$-atom and electron scattering data. However, they do not exclude anomalous effects expected from differences between the muon-nucleus and electron-nucleus interaction ${ }^{2}$ ). An analysis of Rinker and Wilets ${ }^{3}$ ) of the available data in the range $Z=20-30$ places a limit of $\left\langle r^{2}\right\rangle_{\mu}-\left\langle r^{2}\right\rangle_{\mathrm{e}}=-0.01 \pm 0.07 \mathrm{fm}$. However, the lowenergy electron scattering data used for this analysis have been calibrated with the carbon cross section, therefore the comparison is reduced to a comparison of the carbon data only.

All recent elastic electron scattering experiments for carbon are consistent. The results of Jansen et al. ${ }^{6}$ ), Fey et al. ${ }^{7}$ ), Engfer and Türck ${ }^{8}$ ) and the high-energy data of Sick and McCarthy ${ }^{9}$ ) are in excellent agreement, whereas the value found by Bentz ${ }^{10}$ ) is significantly lower. Taking into account a neglected correction for these gas target measurements Schütz ${ }^{7}$ ) found a good agreement of these data too. The sensitivity of the muonic transition energies to the nuclear charge distribution has a strong $Z$-dependence. In order to deduce a charge radius for carbon from muonic $\mathrm{K} \mathrm{X}$-ray transitions with comparable precision of a few percent, the energies have to be determined with an error of $\pm 15 \mathrm{eV}$ or better. Radii deduced from earlier data of muonic carbon, nitrogen and oxygen ${ }^{11}$ ) have an order of magnitude bigger errors than those obtained from electron scattering.

In the present work precise energies of the $\mathrm{K} \mathrm{X}$-rays of muonic carbon, nitrogen and oxygen are determined from which radii are deduced comparable in precision to the low-energy electron scattering data.

\section{Experimental method}

The experiment was performed at the CERN muon channel. The experimental set-up has been described elsewhere ${ }^{12}$ ). Only those special features important for the energy calibration will be discussed here. A V-shaped target was used, which contained $70 \mathrm{~g}$ dimethylglyoxim $\left(\mathrm{C}_{4} \mathrm{H}_{8} \mathrm{~N}_{2} \mathrm{O}_{2}\right)$ and $18 \mathrm{~g}$ rhodium powder. Muonic $\mathrm{Rh} \mathrm{X}$-rays were used for the energy calibration in order to detect shifts between the prompt and calibration spectra. The amount of $\mathrm{Rh}$ was chosen in order to get line intensities comparable to those of the $\mathrm{K}$-series of $\mathrm{C}, \mathrm{N}$ and $\mathrm{O}$. A $\gamma-\gamma$ coincidence between the $\mathrm{Ge}(\mathrm{Li})$ detector and a $12.7 \times 7.2 \mathrm{~cm}^{2} \mathrm{NaI}$ counter allows to distinguish between events from low- $Z$ atoms $(\mathrm{C}, \mathrm{N}, \mathrm{O})$ and from rhodium. The discriminator of the $\mathrm{NaI}$ counter was set above the $3 \mathrm{~d}-2 \mathrm{p}$ transition energy but below the $\mathrm{K}$-series of muonic $\mathrm{C}, \mathrm{N}$ and $\mathrm{O}$, thus inhibiting the appearance of the K-series in the NaI coincident-Ge(Li) spectrum. The coincidence spectra on the other hand contain almost all $\mathrm{X}$-ray transitions of $\mathrm{Rh}$. Hence the coincidence condition reduces their intensities in the $\mathrm{Ge}(\mathrm{Li})$ spectrum to about $30 \%$ only due to the solid angle and efficiency of the $\mathrm{NaI}$ detector, whereas the $\mathrm{C}, \mathrm{N}$ and $\mathrm{O}$ spectra are reduced to about $0.5 \%$ [ref. $\left.{ }^{13}\right)$ ]. The $\mathrm{Ge}(\mathrm{Li})$ detector used was a $1 \mathrm{~cm}^{3}$ planar type with an energy resolution of $600 \mathrm{eV}$ at $120 \mathrm{keV}$. 


\section{Analysis of the data and energy calibration}

A section of the muonic spectrum is shown in fig. 1 summed over the prompt and part of the delayed X-rays. The spectra were analysed as described in ref. ${ }^{12}$ ). Each K-line was fitted with their two fine structure components correlated in position and intensity. A statistical population was assumed for the intensities. Unresolved background lines were taken into account using known energies and intensities correlated to undisturbed transitions.

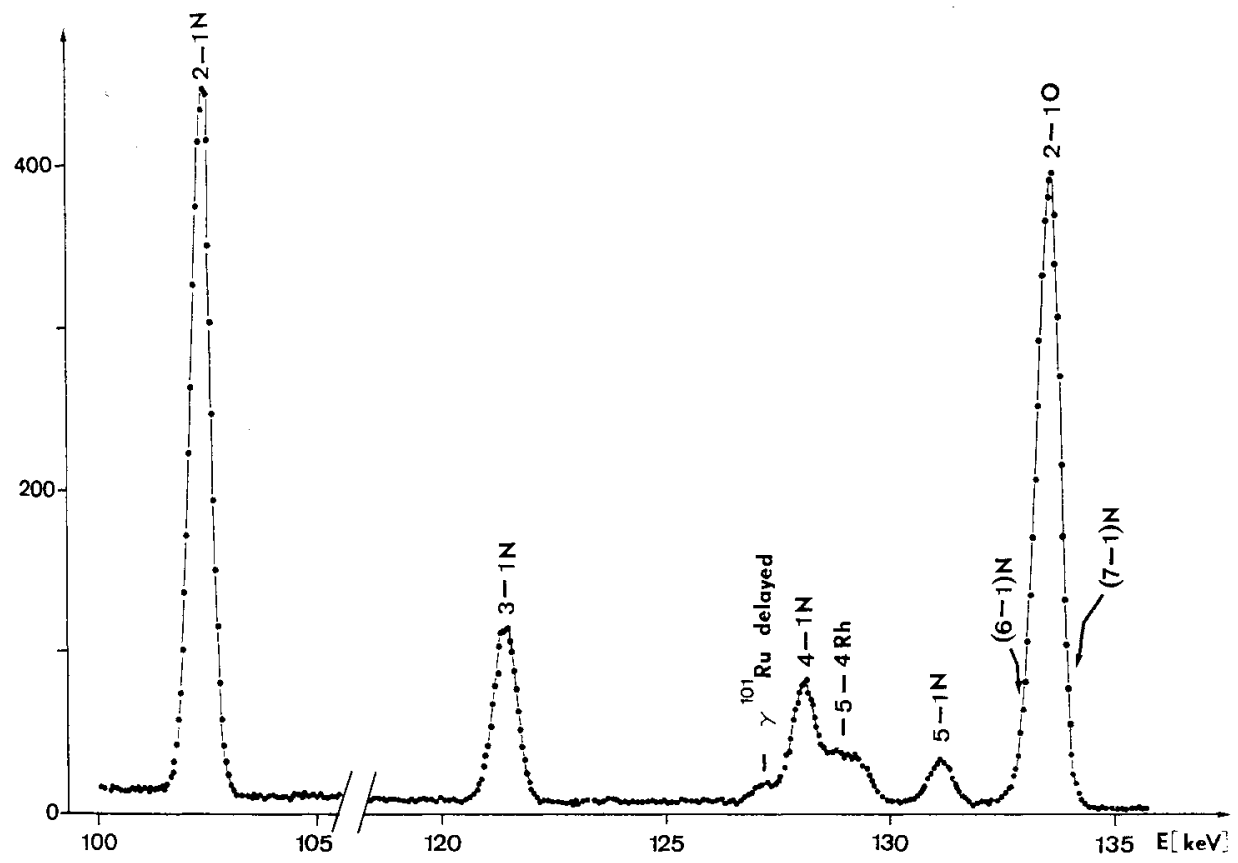

Fig. 1. Part of the $\mu \mathrm{X}$-ray spectrum of the $\mathrm{C}_{4} \mathrm{H}_{8} \mathrm{~N}_{2} \mathrm{O}_{2}+\mathrm{Rh}$ target.

The energy calibration of the spectra were obtained in two steps:

(i) Calibration spectra were measured simultaneously with the muonic spectra ${ }^{12}$ ). A calibration event was accepted during a $250 \mathrm{~ns}$ gate opened by a pulse in a counter no. 0 in the deflected pion beam, provided this gate is not in coincidence with the telescope counters. The use of counter no. 0 gives a time structure for the calibration events similar to that of the muonic X-rays. Radioactive sources of ${ }^{182} \mathrm{Ta}$ and ${ }^{57} \mathrm{Co}$ were used for calibration. Their $\gamma$-ray energies were taken from table 9 of the work of Greenwood et al. ${ }^{14}$ ). The tantalum source was encapsulated in a $1 \mathrm{~cm}^{3}$ plastic scintillator mounted on a photomultiplier. Thus the ${ }^{182} \mathrm{Ta}$ spectrum could be measured with good efficiency in coincidence with the $\beta$-rays from the source yielding a calibration spectrum free of background.

(ii) The second step was to detect possible energy shifts of the calibration spec- 
TABLE 1

Energies of $\mu$ X-rays of ${ }^{103} \mathrm{Rh}$ used as calibration lines

\begin{tabular}{crcr}
\hline Transition & $E_{\text {calc }}(\mathrm{eV})$ & $E_{\text {scr }}(\mathrm{eV})$ & $E_{\text {tota1 }}(\mathrm{eV})$ \\
\hline $7-6$ & 42112 & -48 & 42064 \\
$9-7$ & 46008 & -181 & 45827 \\
$8-6$ & 69424 & -145 & 69279 \\
$6-5$ & 69948 & -33 & 69915 \\
$7-5$ & 111843 & -107 & 111736 \\
$5-4$ & 129127 & -21 & 129106
\end{tabular}

$E_{\text {cale }}$ is the calculated energy of the centre of gravity of the fine structure components including vacuum polarization in first order. The total uncertainties are less than $6 \mathrm{eV}$.

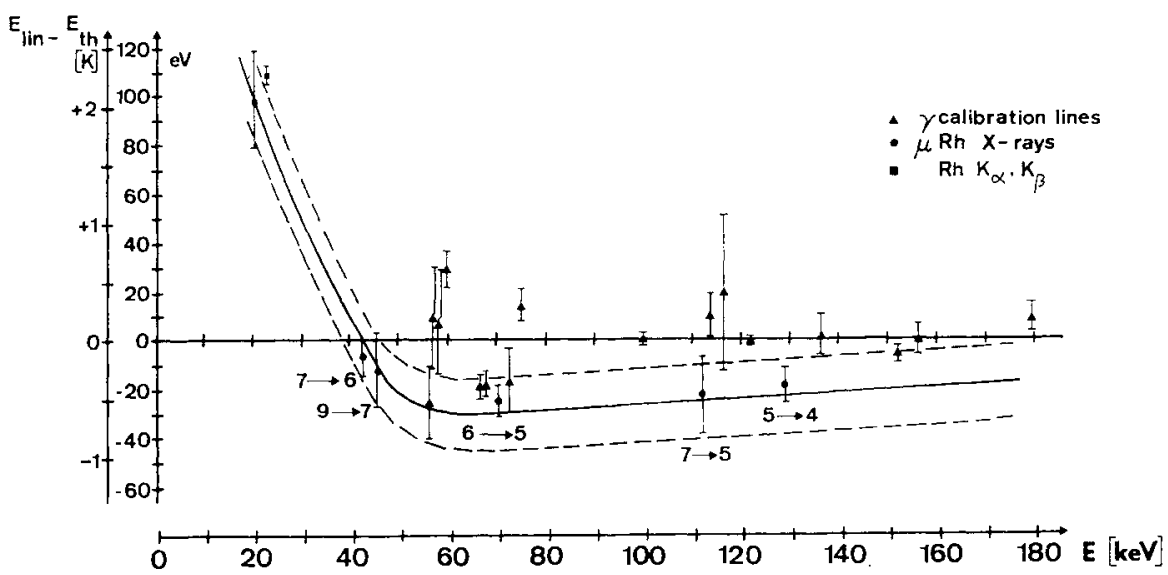

Fig. 2. Nonlinearity of the electronic system. The band of $\pm 15 \mathrm{eV}$ between the dashed lines is determined from the deviation of the rhodium X-ray positions from a linear fit to the $\gamma$-calibration lines.

trum with respect to the prompt one in the energy region of interest $(60-150 \mathrm{keV})$. Such shifts arise from different positions and sizes of the calibration sources and the target relative to the $\mathrm{Ge}(\mathrm{Li})$ counter. For this purpose transitions between higher states in muonic rhodium have been used as calibration lines in the prompt spectrum. The X-ray energies of $\mathrm{Rh}$ in the range from $50-150 \mathrm{keV}$ can be calculated with an accuracy of $10 \mathrm{eV}$ (table 1 ). The finite size effect for circular orbits with $n \geqq 4$ is less than $1 \mathrm{eV}$. Therefore the choice of the parameters of the Fermi charge distribution is not critical. Vacuum polarization to first order in $\alpha$ was calculated using perturbation theory. Higher orders amount to less than $5 \mathrm{eV}$ for $n>4$. Observed differences between the measured and calculated higher quantum electrodynamical corrections ${ }^{15,16}$ ) have no measurable influence on the transitions of interest here. In addition these differences have been reduced by new calculations of higher order vacuum polarization contributions ${ }^{17}$ ). But possible deviations between experiment 
and theory of a few eV may still exist for transition energies below $150 \mathrm{keV}$. The energies given in table 1 correspond to the center of gravity of the unresolved fine structure components. Their intensities were calculated with a cascade program developed by Hüfner ${ }^{18}$ ). The electron screening corrections (table 1) were calculated with a program developed by Vogel ${ }^{19}$ ) assuming all electrons present. Following Vogel's arguments a practically full screening effect is expected.

The nonlinearity curve of the total system is shown in fig. 2 . The difference between the energy calibration from $\gamma$-sources and from the prompt $\mathrm{Rh}$ spectrum amounts to $20 \mathrm{eV}$ maximum and is mainly caused by the different geometry of the calibration sources and the target with respect to the $\mathrm{Ge}(\mathrm{Li})$ detector. The 8-6 and 6-5 transitions are not resolved, therefore, their relative positions and intensities have been correlated. The experimental error of $\pm 15 \mathrm{eV}$ is shown by the band between the dashed lines.

A further test of the consistency of the energy calibration is obtained from delayed ${ }^{101} \mathrm{Ru}$ lines, produced by the reaction ${ }^{103} \mathrm{Rh}(\mu, 2 \mathrm{n} v)^{101} \mathrm{Ru}$. The energies of the two observed $\gamma$-transitions are in good agreement with the values found by Cook and Johns ${ }^{20}$ ):

$\begin{array}{ll}\text { This work } & \text { Cook and Johns }{ }^{20} \text { ) } \\ 127.22 \pm 0.02 & 127.21 \pm 0.05 \\ 184.15 \pm 0.02 & 184.11 \pm 0.05\end{array}$

In a separate experiment delayed nuclear $\gamma$-lines from the ${ }^{103} \operatorname{Rh}(\mu, \mathrm{n} v)^{102} \mathrm{Ru}$ reaction were compared to the same $\gamma$-lines from a radioactive ${ }^{102} \mathrm{Rh}$ source, measured simultaneously to the muon spectra. No energy shifts beyond the statistical errors of $17 \mathrm{eV}$ for energies above $200 \mathrm{keV}$ have been found ${ }^{16,21}$ ).

The experimental X-ray energies for the three $\mu$-atoms are presented in table 2 . The error is calculated from the error of the energy calibration and the statistical error. The latter one is given in brackets.

TABLE 2

Experimental and calculated best fit energies of the $\mathrm{K}$ X-ray series for carbon, nitrogen and oxygen

\begin{tabular}{|c|c|c|c|c|c|c|}
\hline \multirow[b]{2}{*}{ Transition } & \multicolumn{2}{|c|}{$\mathrm{C}$} & \multicolumn{2}{|c|}{$\mathbf{N}$} & \multicolumn{2}{|c|}{$\mathrm{O}$} \\
\hline & $E_{\exp }(\mathrm{eV})$ & $E_{\text {calc }}(\mathrm{eV})$ & $E_{\exp }(\mathrm{eV})$ & $E_{\text {calc }}(\mathrm{eV})$ & $E_{\exp }(\mathrm{eV})$ & $E_{\text {calc }}(\mathrm{eV})$ \\
\hline $2 p-1 s$ & $\begin{array}{l}75248 \\
\pm 15(0.7)\end{array}$ & 75256 & $\begin{array}{l}102406 \\
\pm 15(1.1)\end{array}$ & 102407 & $\begin{array}{l}133525 \\
\pm 15(1.2)\end{array}$ & 133524 \\
\hline $3 p-1 s$ & $\begin{array}{l}89212 \\
\pm 15(1.2)\end{array}$ & 89212 & $\begin{array}{l}121437 \\
\pm 15(2.5)\end{array}$ & 121436 & $\begin{array}{l}158408 \\
\pm 15(2.8)\end{array}$ & 158413 \\
\hline $4 p-1 s$ & $\begin{array}{l}94095 \\
\pm 15(1.7)\end{array}$ & 94094 & $\begin{array}{l}128091 \\
\pm 16(6.2)\end{array}$ & 128091 & $\begin{array}{l}167114 \\
\pm 15(3.1)\end{array}$ & 167117 \\
\hline $5 p-1 s$ & $\begin{array}{l}96355 \\
\pm 16(3.8)\end{array}$ & 96351 & $\begin{array}{l}131167 \\
\pm 17(7.2)\end{array}$ & 131168 & $\begin{array}{l}171144 \\
\pm 16(4.7)\end{array}$ & 171141 \\
\hline $6 \mathrm{p}-1 \mathrm{~s}$ & $\begin{array}{l}97601 \\
\pm 20(13)\end{array}$ & 97574 & & & $\begin{array}{l}173331 \\
\pm 18(10)\end{array}$ & 173323 \\
\hline
\end{tabular}

The errors in brackets are statistical errors only, $n \mathrm{p}-1 \mathrm{~s}$ is the centre of gravity of the fine structure components. 


\section{Determination of the rms radii}

The energies of the muonic levels were calculated with a computer program developed by Acker $^{22}$ ). First order vacuum polarization was taken into account by a perturbation calculation. The difference between a perturbation calculation and an exact calculation by adding the vacuum polarization potential to the Coulomb potential is less than $1 \mathrm{eV}$. The errors on the calculated transition energies due to the errors of the constants ${ }^{\dagger}$ are less than $0.5 \mathrm{eV}$.

With the exception of the electron screening, the corrections to be added to these binding energies have a measurable influence only on the $1 \mathrm{~s}$ level. The contributions of the various corrections are given in table 3 for the $(2 p-1 s)$ transitions. The higher order vacuum polarization corrections were calculated for oxygen with a program developed by Fricke ${ }^{24}$ ) and extrapolated for carbon and nitrogen. The first order Lamb shift was obtained through the formulas given in ref. ${ }^{25}$ ) taking for the Bethe logarithm the values given therein. The second order Lamb shift correction was taken to be $15 \%$ of the first order one ${ }^{26}$ ). Due to the uncertainty of the Bethe logarithm and the use of the point nucleus approximation, the error on the Lamb shift is assumed to be $50 \%$.

TABLE 3

Contributions to the energies of the centre of gravity of the $2 p-1 \mathrm{~s}$ transitions

\begin{tabular}{|c|c|c|c|}
\hline \multirow[t]{2}{*}{ Contribution } & \multicolumn{3}{|c|}{$E(2 p-1 s)(e V)$} \\
\hline & $\overline{\mathrm{C}}$ & $\mathrm{N}$ & 0 \\
\hline Point nucleus & 75287.9 & 102632.9 & 134216.7 \\
\hline Finite size & -404.6 & -775.4 & -1455.2 \\
\hline Vac. pol. 1st order & 371.4 & 548.1 & 761.5 \\
\hline Vac. pol. 2nd, 3rd order & 2.6 & 3.8 & 5.3 \\
\hline Lamb shift ${ }^{a}$ ) & $-\quad 5.4$ & 9.2 & 14.5 \\
\hline Relativ. reduced mass & 0.4 & 0.7 & 1.0 \\
\hline Nuclear polarization & 3.6 & 6.0 & 9.3 \\
\hline Electron screening & $-\quad 0.1$ & $-\quad 0.1$ & - $\quad 0.1$ \\
\hline Total energy & 75256 & 102407 & 133524 \\
\hline Total estimated error & \pm 7 & \pm 10 & \pm 16 \\
\hline
\end{tabular}

a) Including the self-energy, anomalous magnetic moment and $\mu^{+} \mu^{-}$vacuum polarization.

The nuclear polarization correction to the $1 \mathrm{~s}$ level was taken from Cole ${ }^{27}$ ) for oxygen and extrapolated to nitrogen and carbon. It should be accurate within a factor of 2 . The electron screening for oxygen was calculated by Fricke ${ }^{28}$ ) and agrees well with calculations done by Vogel ${ }^{19}$ ). These corrections become more important with increasing main quantum number. For the $3 p-1 s, 4 p-1 s, 5 p-1 s$ and $6 p-1 s$ transitions in oxygen they amount to $0.8,2.3,5.6$ and $10.9 \mathrm{eV}$ respectively, assuming, like for rhodium, all electrons present. The screening from one $\mathrm{K}$-electron alone amounts to about $50 \%$ of these values.

$+m_{\mu} c^{2}=105.660(1) \mathrm{MeV}, \hbar_{c}=197.32891(66) \mathrm{MeV} \cdot \mathrm{fm}^{-1} ; x^{-1}=137.03602(21) ;$ ref. $\left.{ }^{23}\right)$. 


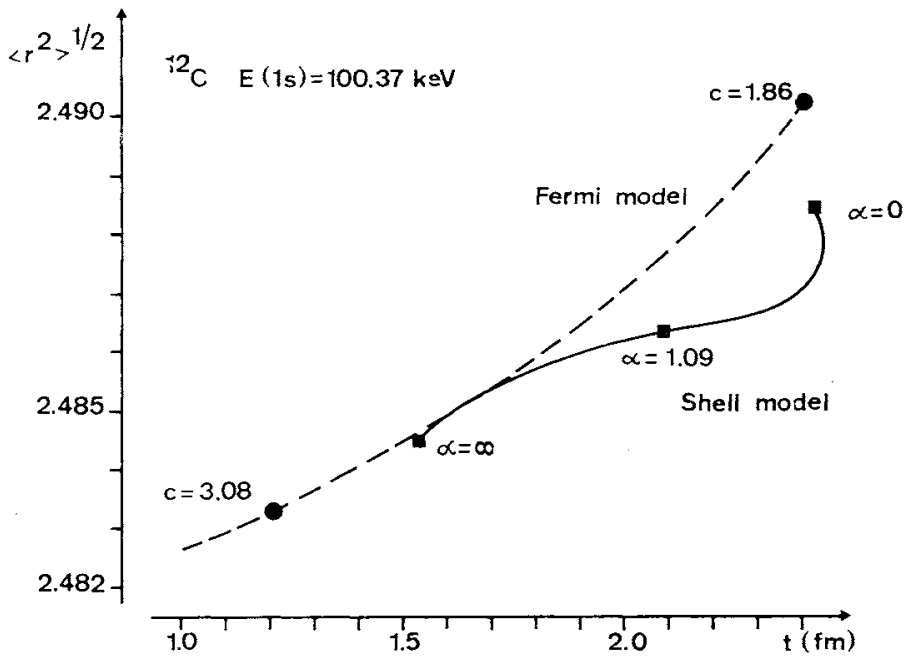

Fig. 3. Dependence of the rms radius on the skin thickness parameter $t$ of the Fermi, dashed curve, and harmonic oscillator shell, full curve, model distribution for carbon. For the shell model $t(a, \alpha)$ has been calculated from the charge distribution as the $90 \%$ to $10 \%$ skin thickness.

The total errors quoted in table 3 for the $2 \mathrm{p}-1 \mathrm{~s}$ transition energies are due to the uncertainties in the Lamb shift and nuclear polarization corrections and are comparable to the experimental ones given in table 2.

The effect of the finite nuclear size can be calculated by assuming some parametrized forms of the nuclear charge distribution. For low- $Z$ elements only the 1 s level has a measurable energy shift due to the finite size, and a nearly model independent rms radius $\left\langle r^{2}\right\rangle^{\frac{t}{2}}$ of the charge distribution can be derived. In fig. 3 the dependence of the carbon rms radius on the skin thickness $t$ is plotted for the two usually assumed charge distributions, the two parameter Fermi distribution

$$
\rho(r)=N\left[1+\exp \left(4 \ln 3 \frac{r-c}{t}\right)\right]^{-1},
$$

with $\rho(c)=\frac{1}{2} N$ and $\rho\left(c \pm \frac{1}{2} t\right)=\left(\frac{1}{2} \mp \frac{2}{5}\right) N$, and the harmonic oscillator shell model distribution

$$
\rho(r)=\rho(0)\left[1+\alpha(r / a)^{2}\right] \mathrm{e}^{-(r / a)^{2}},
$$

taking $a$ and $\alpha$ as free parameters. The two extreme values $\alpha=0$ and $\alpha=\infty$ correspond to Gaussian, and Maxwell distributions respectively. For the shell model the skin thickness $t$ depends on both parameters $a$ and $\alpha$. It has been calculated numerically with $N$ set equal to the maximum value of $\rho(r)$. The rms radius determined from the Fermi distribution shows a stronger model dependence; a variation in $t$ of $25 \%$ leads to a $\Delta\left\langle r^{2}\right\rangle^{\frac{1}{2}} /\left\langle r^{2}\right\rangle^{\frac{1}{2}}=0.1 \%$, whereas the same variation in $t(\alpha)$ of the shell model gives a relative change in the rms radius of $0.04 \%$ only (fig. 3 ).

The harmonic oscillator shell model distribution was assumed for the analysis of 
our data as it is also often used in the calculations of the electron scattering cross sections. In the strict sense of this model the only free parameter which can be fitted to the experimental data is $a$ or the rms radius. From fig. 3 an uncertainty of less than $0.005 \mathrm{fm}$ from the choice of the model can be deduced which is negligible compared to present experimental errors. As another choice of the model the $\mathrm{K}$ series of oxygen was analysed with a three parameter Fermi distribution. The parameter $w$, which is best determined from high-energy (e, e) scattering data, as well as $t$ were taken from ref. ${ }^{9}$ ). We obtain an $0.002 \mathrm{fm}$ greater rms radius than with the shell model charge distribution. A model independent analysis of the $2 \mathrm{p}-1 \mathrm{~s}$ and $3 \mathrm{p}-1 \mathrm{~s}$ transitions with the generalized moment $\left\langle\mathrm{e}^{-\alpha r} r^{k}\right\rangle$ defined by Barrett ${ }^{29}$ ) yields equivalent radii for carbon, nitrogen and oxygen respectively of: $R_{k}(\mathrm{C})=$ $3.22 \pm 0.06 \mathrm{fm}$ with $k=2.113$ and $\alpha=0.045 ; R_{k}(\mathrm{~N})=3.28 \pm 0.04$ with $k=2.112$ and $\alpha=0.047$; and $R_{k}(\mathrm{O})=3.48 \pm 0.02$ with $k=2.112$ and $\alpha=0.048$.

For each transition of the $\mathrm{K}$-series a rms radius was determined. A mean value was then calculated for each element, taking into account the error from the line fit only. The energies calculated with this rms radius, using the shell model parameters $a$ and $\alpha$ given in table 4 are tabulated in table 2 together with the experimental energies. The agreement between measured and calculated values is very good, indicating that no additional systematic errors are present.

The rapid decrease of the sensitivity $\Delta E / \Delta\left\langle r^{2}\right\rangle$ with decreasing $Z$ is illustrated by the values given in column 3 of table 4 . Tabulated is the reciprocal value of the sensitivity of the $1 \mathrm{~s}$ level, which is a constant over a rather wide range of $\left\langle r^{2}\right\rangle$ and $\triangle E$.

TABLE 4

Best fit parameters of the charge distribution; $a$ and $\alpha$ are the shell model parameters

\begin{tabular}{lcccr}
\hline & $\left\langle r^{2}\right\rangle\left(\mathrm{fm}^{2}\right)$ & $\left\langle\left\langle r^{2}\right\rangle / \Delta E_{1 \mathrm{~s}}\left(\mathrm{fm}^{2} / \mathrm{eV}\right)\right.$ & $a(\mathrm{fm})$ & $\alpha$ \\
\hline $\mathrm{C}$ & $6.19 \pm 0.24(0.10)$ & 0.016156 & 1.708 & 1.092 \\
$\mathrm{~N}$ & $6.30 \pm 0.13(0.10)$ & 0.008971 & 1.735 & 1.295 \\
$\mathrm{O}$ & $7.32 \pm 0.08(0.09)$ & 0.005475 & 1.826 & 1.532 \\
\hline
\end{tabular}

The errors of $\left\langle r^{2}\right\rangle$ are experimental ones, the uncertainty due to theoretical corrections is given in brackets. $\Delta\left\langle r^{2}\right\rangle / \Delta E_{1 \mathrm{~s}}$ is the reciprocal value of the sensitivity of the $1 \mathrm{~s}$ level.

\section{Discussion}

The rms radii obtained in the present work are tabulated in table 5. The errors include experimental and theoretical ones. As can be seen from table 5 the agreement between the present results and the elastic electron scattering data is very good. Differences between muonic and electronic interactions, therefore, are smaller than the present experimental accuracy. Also, from a recent elastic muon scattering experiment no such differences are observed ${ }^{30}$ ). From the data of table 5 an upper 
TABLE 5

Comparison of recent $\mathrm{rms}$ radii deduced from elastic electron scattering (e, e) and muonic atoms

\begin{tabular}{|c|c|c|c|c|}
\hline & \multicolumn{4}{|c|}{$\left\langle r^{2}\right\rangle \frac{1}{2}(\mathrm{fm})$} \\
\hline & ${ }^{12} \mathrm{C}$ & $14 \mathrm{~N}$ & ${ }^{16} \mathrm{O}$ & Ref. \\
\hline$(\mathrm{e}, \mathrm{e}) ; 20-80 \mathrm{MeV}$ & $\left.2.453 \pm 0.008^{a}\right)$ & & & $\left.{ }^{6}\right)$ \\
\hline$(\mathrm{e}, \mathrm{e}) ; 400+750 \mathrm{MeV}$ & $\left.2.46 \pm 0.025^{b}\right)$ & & $\left.2.73 \pm 0.025^{c}\right)$ & ${ }^{9}$ ) \\
\hline$(\mathrm{e}, \mathrm{e}) ;$ model-indep. & $\left.2.468 \pm 0.016^{\mathrm{d}}\right)$ & & & 31) \\
\hline$(\mathrm{e}, \mathrm{e}) ; 30-60 \mathrm{MeV}$ & $2.462 \pm 0.022$ & $2.54 \pm 0.02$ & $2.718 \pm 0.021$ & $\left.{ }^{7}\right)$ \\
\hline$(\mu, \mu) ; 78 \mathrm{MeV}$ & $\left.2.32 \pm 0.16^{\mathrm{a}}\right)$ & & & 29) \\
\hline$\mu$ & $2.40 \pm 0.56$ & $2.67 \pm 0.26$ & $2.61 \pm 0.14$ & 11) \\
\hline$\mu$ & $2.49 \pm 0.05$ & $2.55 \pm 0.03$ & $2.71 \pm 0.02$ & present work ${ }^{e}$ ) \\
\hline
\end{tabular}

The energy range of the $(e, e)$ experiments is given.

i) Model dependent fit with a harmonic oscillator shell model charge distribution.

b) Modified shell model charge distribution.

c) Modified three parameter Fermi distribution.

d) Model independent analysis of the combined data of refs. ${ }^{6,9}$ ).

e) The errors include uncertainties of the theoretical corrections.

limit of $\left\langle r^{2}\right\rangle_{\mu}-\left\langle r_{\mathrm{e}}^{2}\right\rangle\langle 0.17 \mathrm{fm}$ can be deduced. A drawback of the analysis of muonic and $(\mathrm{e}, \mathrm{e})$ data is the use of model distributions for the charge density $\rho(r)$. The error bars of the deduced rms radii do not include the model dependence and therefore, are generally underestimated. A model-independent phenomenological charge density and rms radius was derived for carbon (third row of table 5) by a common analysis of high ${ }^{9}$ ) and low ${ }^{6}$ ) momentum transfer $(e, e)$ scattering data by Sick ${ }^{31}$ ). An inclusion of the muonic data in Sick's model-independent analysis would not improve the results, because of the still higher precision of the (e, e) data. An increase of the accuracy of future muonic atom experiments implies also better theoretical calculations for the different corrections mentioned in sect. 4. This may be best pointed out by the fact that the finite size effect in carbon is of the same order of magnitude as the first-order vacuum polarization correction.

The authors wish to thank Drs. R. Link, R. Michaelsen, W. U. Schröder and B. Robert-Tissot for their help during the experiments and Prof. L. Schaller for useful discussions. This work was supported by the Bundesministerium für Bildung und Wissenschaft and the Schweizerische Nationalfonds.

\section{References}

1) R. Engfer, Proc. Int. School of Physics Enrico Fermi, Varenna, 1966

2) S. Barshay, Phys. Lett. 37B (1971); Phys. Rev. D7 (1973) 2635

3) G. A. Rinker, Jr. and L. Wilets, Phys. Rev. D7 (1973) 2629

4) J. Bailey and E. Picasso, Prog. Nucl. Phys. 12 (1970) 43

5) S. J. Brodsky and S. D. Drell, Ann. Rev. Nucl. Sci 20 (1970) 147

6) J. A. Jansen, R. Th. Peerdeman and C. de Vries, Nucl. Phys. A188 (1972) 337 
7) G. Fey, H. Frank, W. Schütz and H. Theissen, Z. Phys. 265 (1973) 401; W. Schütz, Inst. f. Kernphysik, T. H. Darmstadt, thesis, to be published

8) R. Engfer and D. Türck, Z. Phys. 205 (1967) 90

9) I. Sick and J. S. McCarthy, Nucl. Phys. A150 (1970) 631

10) H. A. Bentz, Z. Phys, 243 (1971) 153

11) G. Backenstoss, S. Charalambus, H. Daniel, H. Koch, G. Poelz, H. Schmitt and L. Tauscher, Phys. Lett. 25B (1967) 547

12) H. Backe, R. Engfer, U. Jahnke, E. Kankeleit, R. M. Pearce, C. Petitjean, L. Schellenberg, H. Schneuwly, W. U. Schröder, H. K. Walter and A. Zehnder, Nucl. Phys. A189 (1972) 472

13) H. K. Walter, H. Backe, R. Engfer, E. Kankeleit, R. Link, R. Michaelsen, C. Petitjean, L. Schellenberg, H. Schneuwly, W. U. Schröder, J. L. Vuilleumier and A. Zehnder, Nucl. Phys., to be published

14) R. C. Greenwood, R. G. Helmer and R. J. Gehrke, Nucl. Instr. 77 (1970) 141

15) M. S. Dixit, H. L. Anderson, C. K. Hargrove, R. J. McKee, D. Kessler, H. Mes and A. C. Thompson, Phys. Rev. Lett. 27 (1971) 878

16) H. K. Walter, J. L. Vuilleumier, H. Backe, F. Boehm, R. Engfer, A. H. von Gunten, R. Link, R. Michaelsen, C. Petitjean, L. Schellenberg, H. Schneuwly, W. U. Schröder and A. Zehnder, Phys. Lett. 40B (1972) 197

17) J. Blomquist, Nucl. Phys. B48 (1972) 95;

M. K. Sundaresan and P. J. S. Watson, Phys. Rev. Lett. 29 (1972) 15;

T. C. Bell, Phys. Rev. A7 (1973) 1480

18) J. Hüfner, Z. Phys. 195 (1966) 365

19) P. Vogel, Phys. Rev. A7 (1973) 63; preprint Calt. 63-188

20) B. Cook and M. W. Johns, Can. J. Phys. 50 (1972) 1957

21) J. L. Vuilleumier et al., to be published

22) H. L. Acker, G. Backenstoss, C. Daum, J. C. Sens and S. A. de Wit, Nucl. Phys. 87 (1966) 1

23) Particle Data Group, Rev. Mod. Phys. 43 (1971) 51

24) B. Fricke, Z. Phys. 218 (1969) 495

25) H. A. Bethe and E. E. Salpeter, Handbuch der Physik 35 (1957) 88

26) R. C. Barrett, Phys. Lett. 28B (1968) 93

27) R. K. Cole, Phys. Rev. 177 (1968) 164

28) B. Fricke, Nuovo Cim. Lett. 2 (1969) 859

29) R. C. Barrett, Phys. Lett. 33B (1970) 388

30) T. Sanford, S. Childress, G. Dugan, L. M. Ledermann and L. E. Price, Phys. Rev. C8 (1973) 896

31) I. Sick, Phys. Lett. 44B (1973) 62; Nucl. Phys., in press 